\title{
Vessel Reconstruction in Acute Basilar Artery Occlusion
}

\author{
Kellie Davis, Michael Curran, Cheemun Lum
}

Can. J. Neurol. Sci. 2010; 37: 135-137

Twenty percent of ischemic events involve the posterior circulation..$^{1-4}$ Basilar artery (BA) occlusions carry an extremely high risk of disability and mortality. ${ }^{1-4}$ Effective management of these acute strokes demands prompt treatment and knowledge of various recanalization strategies. Acute interventions can include mechanical disruption by angioplasty, clot retrieval or stent placement in combination with thrombolytic and antiplatelet medications.

The following case report describes use of various recanalization strategies to treat a significant amount of BA thrombosis with associated dominant right vertebral artery (RVA) origin occlusion in a 52-year-old female patient. The BA and RVA were successfully recanalized and reconstructed with a Neuroform and Express (Boston Scientific, Natick, MA) stent, respectively, resulting in excellent antegrade flow through the vertebrobasilar system.

\section{Case Report}

A 52-year-old female presented to the emergency department after developing weakness of the left leg and subsequently collapsing. Following collapse, she then developed left-sided facial droop, left eye ptosis, slurred speech and left hemiplegia. Upon arrival, the patient exhibited severe dysarthria but was alert and oriented. Neurological examination revealed the pupils were equal and reactive to light and accommodation with evoked nystagmus on left gaze. Mild weakness was demonstrated on the left side and reflexes were brisk with flexor plantar responses. The remainder of the examination was unremarkable. The past medical history included hypertension and smoking.

A CT angiogram revealed an occlusive thrombus in the distal $1 \mathrm{~cm}$ of the BA; the proximal RVA at its origin was occluded with thrombus (Figures 1 and 2). The left vertebral artery originated from the aortic arch and was significantly hypoplastic. Neurology and neuroradiology were consulted and the patient was consented for neuro-interventional management. The patient was intubated.

An angiographic run revealed complete occlusion at the origin of the RVA. The RVA was reconstituted in the midcervical portion with collaterals, however flow was extremely sluggish distally with delayed BA opacification. Two thousand units of heparin were administered. The expected origin of the RVA was gently probed with a Glidewire (Terumo Medical, Somerset, NJ) until it was easily advanced distal to the occlusion. The diagnostic catheter was advanced above the level of occlusion.

Catheter angiography (not shown) revealed a large amount of thrombus in the distal RVA at the vertebral basilar junction and

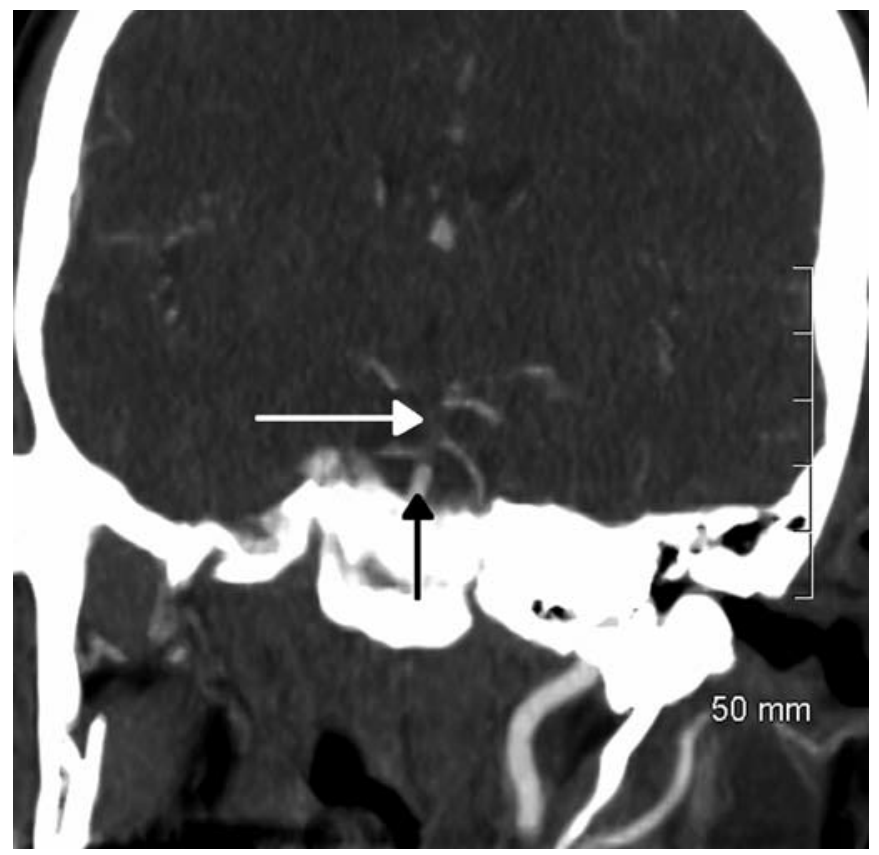

Figure 1: CT angiogram, coronal. Proximal patent basilar artery (black arrow) with distal occlusion (white arrow).

no BA flow. An exchange wire was used to maneuverer an $8 \mathrm{Fr}$ Mechanical Embolus Removal in Cerebral Ischemia (MERCI) (Concentric Medical, Mountainview, CA) balloon guiding catheter above the proximally occluded RVA.

An 18 MERCI microcatheter was advanced over an Xpedion 14 wire through the BA clot into the left P1 segment under road map guidance. Three passes were performed with a MERCI X 5 retriever with a modest amount of visible clot verified on the retriever. This resulted in recanalization of the distal RVA and proximal third of the BA.

From the Head Interventional and Diagnostic Neuroradiology (CL), Faculty of Medicine (KD), Department of Anesthesia (MC), University of Ottawa, The Ottawa Hospital, Ottawa, ON, Canada.

Received April 23, 2009. Final Revisions Submitted July 10, 2009. Correspondence to: Cheemun Lum, Head-Interventional and Diagnostic Neuroradiology, University of Ottawa, The Ottawa Hospital - Civic Campus, 1053 Carling Avenue, Ottawa, Ontario, K1Y 4E9, Canada. 


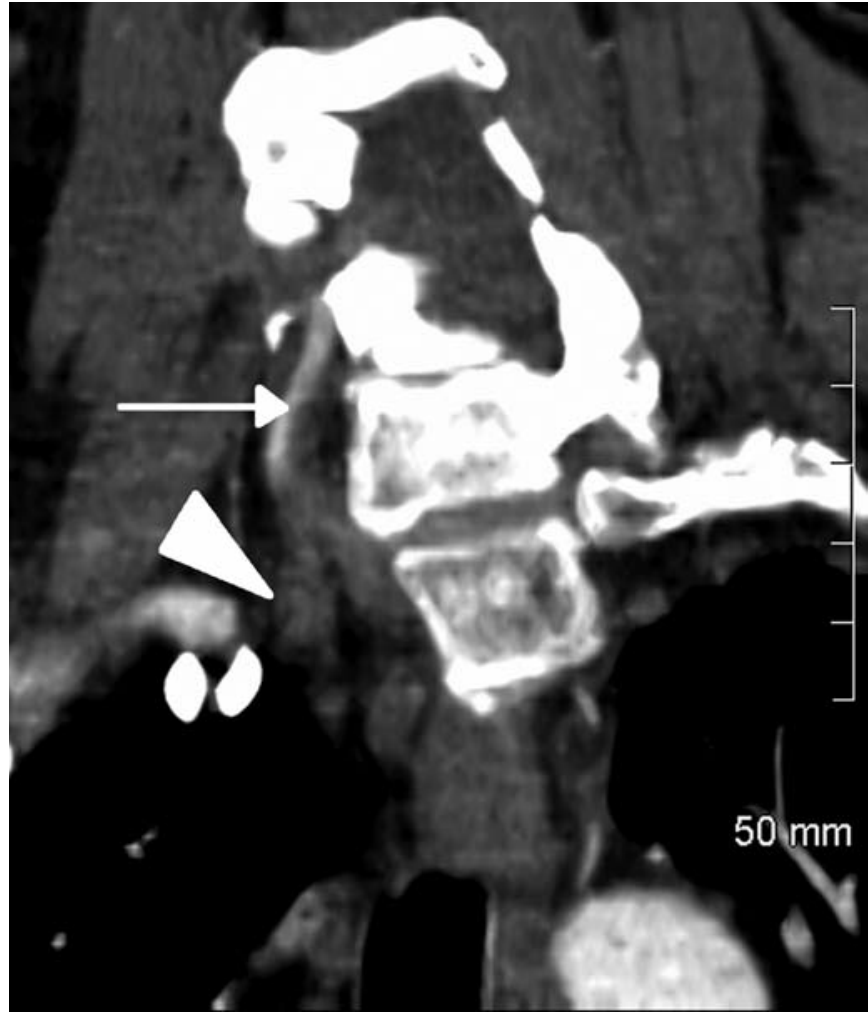

Figure 2: CT angiogram, coronal. The dominant right vertebral artery (arrow) was occluded by a clot (arrowhead) at its origin.

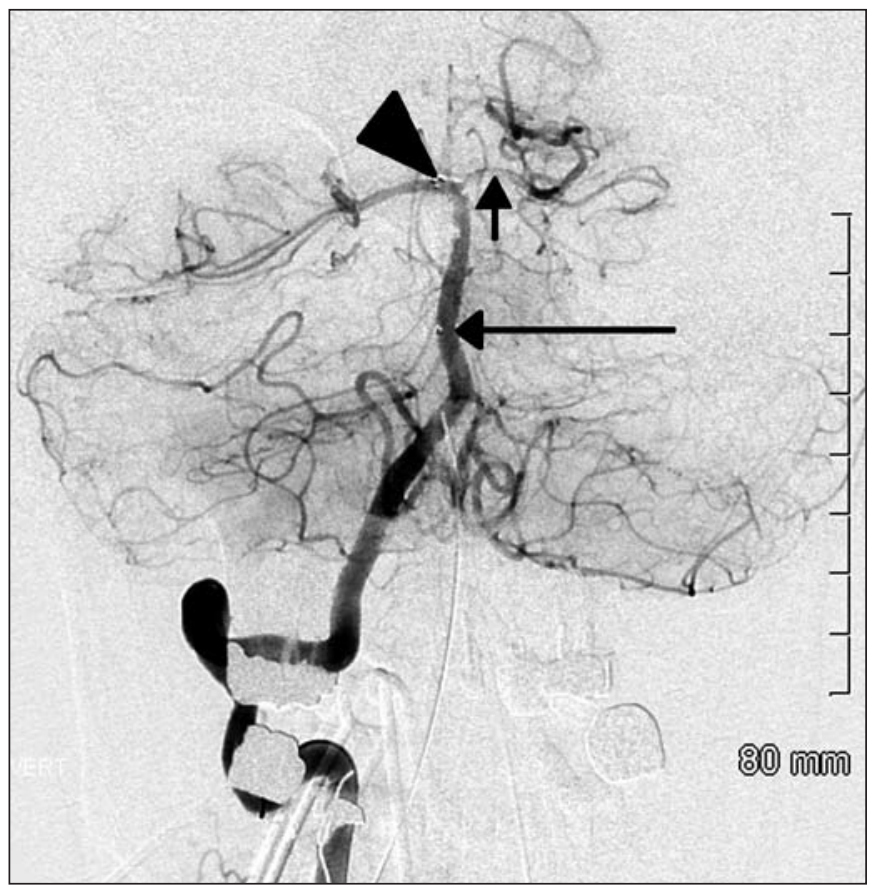

Figure 3: Angiogram, cerebral. Post-deployment of Neuroform stent showing recanalized basilar artery. Distal (arrowhead) and proximal (long arrow) stent markers. Residual clot in the left posterior cerebral artery (short arrow).
Angioplasty of the residual BA clot was performed multiple times with a 4 X 15 mm Hyperglide (Microtherapeutics, Irvine, CA) balloon resulting in partial intermittent BA flow. Unfortunately, there was recurrent thrombosis of the distal BA. Fifteen milligrams of tPA was infused intra-arterially with no significant recanalization. A nasogastric tube was placed by the anesthetist into the stomach and the patient was loaded with 300 $\mathrm{mg}$ of Plavix and $325 \mathrm{mg}$ of Aspirin.

A $3.5 \times 20 \mathrm{~mm}$ Neuroform stent was then successfully deployed into the BA extending into the P1 segment (Figure 3) resulting in immediate restoration of brisk BA flow. A small amount of focal clot remained in the left P1 segment. A check angiogram performed through the guiding catheter demonstrated poor washout of the RVA suggesting persistent significant proximal occlusion. The guiding catheter was retracted into the innominate artery. An angiography run demonstrated 80\% RVA stenosis with a focal amount of clot seen at the origin.

An 8 French Cook (Cook Medical, Bloomington, IN) $70 \mathrm{~cm}$ sheath was placed at the origin of the RVA. Following this, a balloon-mounted Express (Boston Scientific, Natick, MA) 4 x 15 $\mathrm{mm}$ stent was deployed. Brisk flow through the RVA and BA stent was monitored for 20 minutes prior to procedure termination. A Dyna CT performed in the angiography suite revealed no obvious hemorrhage.

A post-operative MRI Brain revealed several small infarcts bilaterally in the superior cerebellar artery territory and right hemipons. Patency was successfully restored to the basilar and proximal RVA (Figure 4). Clinically, the patient was dysarthric with a left-sided facial droop and strength of $3+/ 5$ in all extremities.

During the course in hospital, the patient had an impressive recovery with improvement of all neurological deficits. Upon discharge to rehabilitation, strength had improved to $4+/ 5$ in the

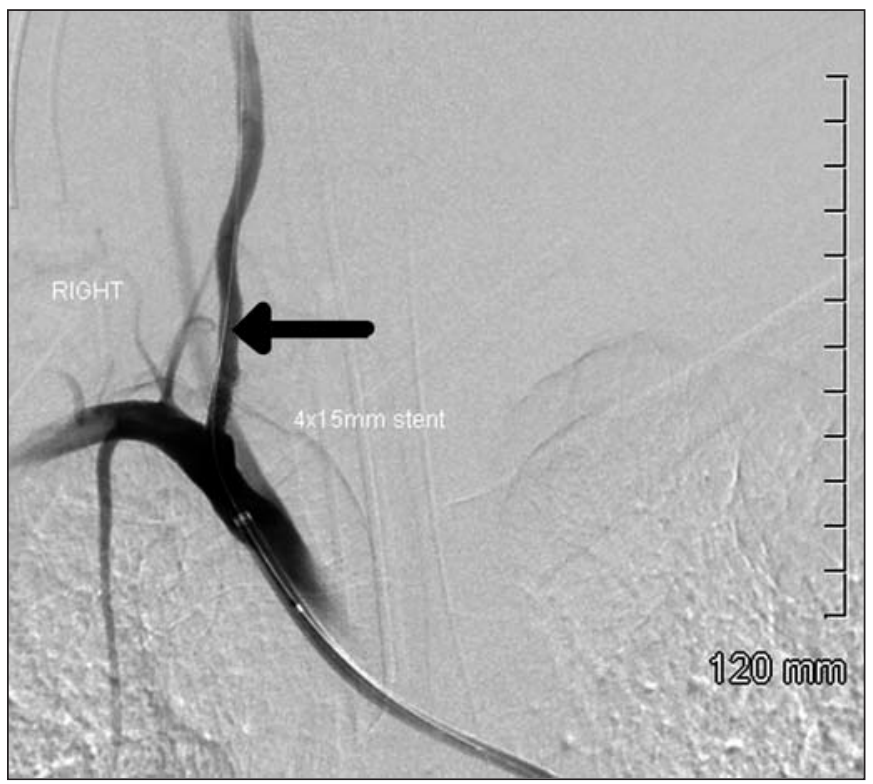

Figure 4: Post-stenting AP of the patent right vertebral artery (arrow). 
left limbs with a left pronator drift. The cranial nerve examination was unremarkable aside from a seventh nerve palsy. The patient was able to walk independently and the dysarthria had nearly resolved.

At three months, the patient was doing well with a modified Rankin score of 1 . Minimal left arm and left leg weakness and slight dysarthria remained, however she was able to carry out all activities of daily living without assistance.

\section{Discussion}

This case illustrates the use of multiple techniques to recanalize an acutely occluded BA. We chose to address the BA thrombus first, as we feel that in cases of tandem clots, the symptoms are caused primarily by the intracranial clot and should be the initial focus. ${ }^{2}$ There was significant clinical concern about her low-flow state given the occluded dominant RVA, hypoplastic left vertebral artery (LVA) and BA clot.

It is at times difficult to find the expected lumen, especially when the artery is completely occluded. In many cases, the clot can be traversed, however this depends on its consistency. One other potential risk is of entering the false lumen of a dissection. To avoid this, gentle probing with a curved microwire without pushing against resistance is advised to increase the likelihood of entering the true lumen. ${ }^{5}$ There is also a possibility of dislodging clot, which can embolize distally. Once through the occluded vertebral artery, several techniques were employed to achieve BA recanalization.

The first attempt was with a MERCI X5 retriever, which has been shown to improve outcomes and reduce mortality. ${ }^{6}$ Revascularization of vertebrobasilar artery (VBA) clots occurs at rates as high as $78 \%$, leading to modified Rankin Scores of 0-2 in $33 \%$ of cases. ${ }^{7}$ As reported in the MERCI trials, this treatment is an option up to eight hours after an acute stroke for patients where thombolytics are contraindicated. ${ }^{6}$

Thrombolytics alone are typically insufficient in treating VBA clots, as the clot burden tends to be high. ${ }^{6,8}$ A combination of tissue plasminogen activator (tPA) and mechanical thrombolysis may lead to superior results despite the theoretical concern of hemorrhage when combining the two. However, the use of both MERCI and tPA have not been found to increase the risk of hemorrhage or serious complication. ${ }^{9}$ Conversely, MERCI retriever use may actually reduce the need of increasing doses of thrombolytics, further reducing the risk of bleeding related complications. ${ }^{10}$

Perforation is a concern when using MERCI retrievers. This risk could theoretically be increased when making multiple passes with the device, as is often necessary. However, the rate of perforation has been reported as low as $3.8 \%$ and did not occur during this procedure. ${ }^{9}$

Second and third attempts at revascularization were made with angioplasty followed by intra-artial tPA. Angioplasty has been successfully performed in cases of acute stroke. ${ }^{11-14}$ However, in this case, the thrombus was refractory to both treatments.

Successful acute stenting of intracranial clot has been described in the literature. ${ }^{15,16}$ In preparation for placement of stents in both the basilar and RVA, the patient was loaded with Plavix and ASA to prevent acute in-stent thrombosis. ${ }^{13}$ After deployment of the BA Neuroform stent, the BA was patent, however, inflow through the proximally occluded RVA was sluggish. For this reason, the RVA proximally was also stented, resulting in brisk restoration of flow. The flow was angiographically monitored to ensure continued patency prior to withdrawing the guiding catheter.

Acute BA thrombosis carries with it significant morbidity. In the setting of a proximally occluded dominant vertebral artery, we were concerned about further progression of clot and symptoms in a young, previously healthy patient. Dual stents were successfully deployed to recanalize the BA and restore patency in the RVA. In certain cases, multi-modality treatment of acute clot can be helpful.

\section{REFERENCES}

1. Savitz SI, Caplan LR. Vertebrobasilar disease. N Engl J Med. 2005; 352:2618-26.

2. Caplan LR. Posterior circulation disease: clinical findings, diagnosis, and management. Cambridge Mass: Blackwell Sciences; 1996.

3. Voetsch B, Dewitt LD, Pessin MS, Caplan LR. Basilar artery occlusive disease in the New England Medical Center posterior circulation registry. Arch Neurol. 2004;61:496-504.

4. Glass TA, Hennessey PM, Pazdera L. Outcome in thirty days in the New England Medical Center posterior circulation registry. Arch Neurol. 2002;59:369-76.

5. Srinivasan A, Goyal M, Stys P, Sharma M, Lum C. Microcatheter navigation and thrombolysis in acute symptomatic cervical internal carotid occlusion. AJNR Am J Neuroradiol. 2006;27: 774-9.

6. Smith WS, Sung G, Saver J, Budzik R, Duckwiler G, Liebeskind DS, et al. Mechanical thrombectomy for acute ischemic stroke: final results of the Multi MERCI trial. Stroke. 2008;39(4): 1205-12.

7. Lutsep HL, Rymer MM, Nesbit GM. Vertebrobasilar revascularization rates and outcomes in the MERCI and multiMERCI trials. J Stroke Cerebrovasc Dis. 2008;17(2):55-7.

8. Barreto AD, Albright KC, Hallevi H, Grotta JC, Noser EA, Khaja AM, et al. Thrombus burden is associated with clinical outcome after intra-arterial therapy for acute ischemic stroke. Stroke. 2008;39(12):3231-5

9. Smith WS. Safety of mechanical thrombectomy and intravenous tissue plasminogen activator in acute ischemic stroke. Results of the Multi MERCI trial, part I. AJNR Am J Neuroradiol. 2006; 27:1177-82.

10. Levy EI, Ecker RD, Horowitz MB, Gupta R, Hanel RA, Sauvageau $\mathrm{E}$, et al. Stent-assisted intracranial recanalization for acute stroke: early results. Neurosurgery. 2006;58(3):458-63.

11. Lum C, Stys PK, Hogan MJ, Nguyen TB, Srinivasan A, Goyal M. Acute anterior circulation stroke: recanalization using clot angioplasty. Can J Neurol Sci. 2006;33(2):217-22.

12. Yoneyama $\mathrm{T}$, Nakano $\mathrm{S}, \mathrm{Kawano} \mathrm{H}$, Iseda T, Ikeda $\mathrm{T}$, Goya $\mathrm{T}$, et al. Combined direct percutaneous transluminal angioplasty and low dose native tissue plasminogen activator therapy for acute embolic middle cerebral artery trunk occlusion. AJNR Am J Neuroradiol. 2002;23:277-81.

13. Ringer AJ, Qureshi AI, Fessler RD, Guterman LR, Hopkins LN. Angioplasty of intracranial occlusion resistant to thrombolysis in acute ischemic stroke. Neurosurgery. 2001;48:1282-8.

14. Mori T, Kazita K, Mima T, Mori K. Balloon angioplasty for embolic total occlusion of the middle cerebral artery and ipsilateral carotid stenting in an acute stroke stage. AJNR Am J Neuroradiol. 1999;20:1462-4.

15. Fitzsimmons BFM, Becske T, Nelson PK. Rapid stent-supported revascularization in acute ischemic stroke. AJNR Am J Neuroradiol. 2006;27:1132-4.

16. Kelly ME, Furlan AJ, Fiorella D. Recanalization of an acute middle cerebral artery occlusion using a self-expanding, reconstrainable, intracranial microstent as a temporary endovascular bypass. Stroke. 2008;39:1770-3. 